\title{
Focused Ion Beam Direct Write Nanofabrication of Surface Phonon Polariton Metamaterial Nanostructures
}

\author{
N. D. Bassim ${ }^{1}$, A. Giles ${ }^{1}$, J. D. Caldwell ${ }^{1}$, and L. E. Ocola ${ }^{2}$ \\ ${ }^{1}$ Naval Research Laboratory, Code 6366, 4555 Overlook Ave. SW, Washington, DC 20375, USA \\ ${ }^{2}$ Argonne National Laboratory, Argonne, IL 60439, USA
}

Beyond the capabilities for sample characterization via TEM liftout and 3-D sectioning, the focused ion beam microscope (FIB) is useful tool for direct-write lithography of novel materials structures. It has unique control in varying the depth of structure feature through beam and dose control. Because of this, FIB may be used to exploit 3-D resonant modes in metamaterial nanostructures in ways that conventional lithography may not.

In this study, we developed a protocol for patterning arrayed nanostructures that support surface phonon polariton (SPhP) modes supported in polar dielectric materials, such as $\mathrm{SiC}$ and hexagonal-BN. These materials are attracting interest as promising alternatives to plasmonic media, due to the long scattering lifetimes (low optical losses) associated with phonons in polar dielectrics in comparison to free carriers in metals and doped semiconductors. This results in exceptionally narrow resonance linewidths, long propagating polariton modes and high efficiency metamaterials, albeit in the spectral range between the mid-IR to the single digit THz. Recently we have experimentally demonstrated record quality factors from e-beam patterned localized SPhP resonators in $6 \mathrm{H}-\mathrm{SiC}$ nanopillar arrays that were 50-200x smaller than the free-space wavelength. [1] More recent efforts have focused on hexagonal BN (hBN), which is a unique material system that due to the high degree of anisotropy resulting from its polar nature and graphitic planar stacking, results in two spectral bands where the material simultaneously exhibits both metallic and dielectric optical response. Such materials are referred to as hyperbolic metamaterials (HMMs) and are the basis of several optical phenomena such as negative refraction and super-resolution imaging. In order to obtain localized resonance responses, arrays of holes or pillars are necessary to fabricate to achieve high field concentrations.

We present novel metamaterial structures written in $4 \mathrm{H}-\mathrm{SiC}$ and $\mathrm{hBN}$ using an FEI Dual Beam Novalab 6 with a Raith Elphy pattern generator. Prior to milling, we deposited a $40 \mathrm{~nm}$ PMMA resist followed by a $40 \mathrm{~nm} \mathrm{Cr}$ hard mask on the surface in order to mitigate beam tail effects following the technique of Menard et al. [2]. We explored the effects of ion-assisted etching, using precursor gases such as $\mathrm{XeF}_{2}$, water, and iodine-based etchants. Figure 1 is an SEM cross-section on a $\mathrm{XeF}_{2}$-etched array of $200 \mathrm{~nm}$ holes with $600 \mathrm{~nm}$ pitch showing vertical sidewalls and the mitigating effects of the $\mathrm{Cr}$ on reducing beam tailing. We found that water, $\mathrm{XeF}_{2}$, and iodine-based etchants all had increased milling rates over $\mathrm{Ga}$ ions alone, while not affecting the $\mathrm{Cr}$ hard mask. This allowed for columniation of the Cr mask while still achieving reasonable milling rates.

To explore the prospects of polarizability of these SPhPs, we also devised strategies to fabricate chiral metamaterial structures with a polarization dependence using the unique capabilities of the FIB. The ability of the FIB to combinatorially write varying structures and choose the optimal configuration for writing enabled the development of a smooth-sloped structure. Figure 2 shows an array of decreasing-slope chiral array in $4 \mathrm{H}-\mathrm{SiC}$. We also performed such lithography on h-BN structures. 
Use of the Center for Nanoscale Materials was supported by the U. S. Department of Energy, Office of Science, Office of Basic Energy Sciences, under Contract No. DE-AC02-06CH11357

\section{References:}

[1] Caldwell, J.D., et al., Nano Letters. 13(8): p. 3690-3697.

[2] Menard, L., et al., Nano Letters 11(20): p. 512-517.

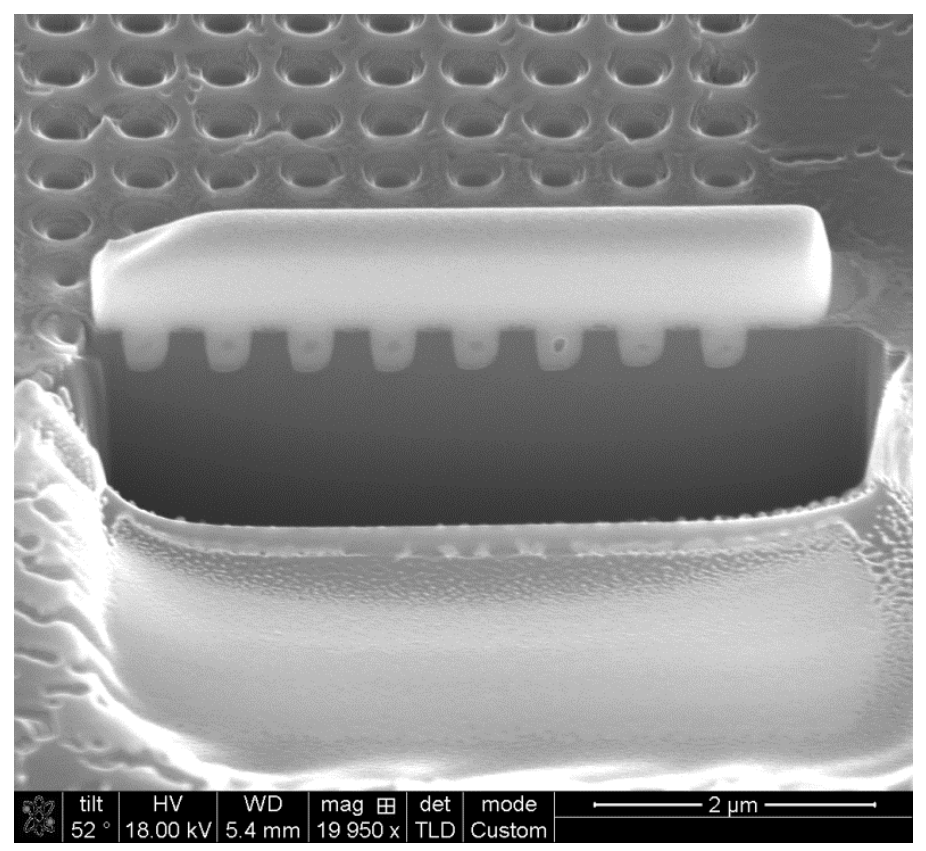

Figure 1: SEM image of $\mathrm{XeF}_{2}$ gas-assisted FIB-etched 200nm circular array through $\mathrm{Cr}$ hard mask after Pt-deposition and cross sectioning showing parallel sidewalls. Sample is tilted at 52 degrees.

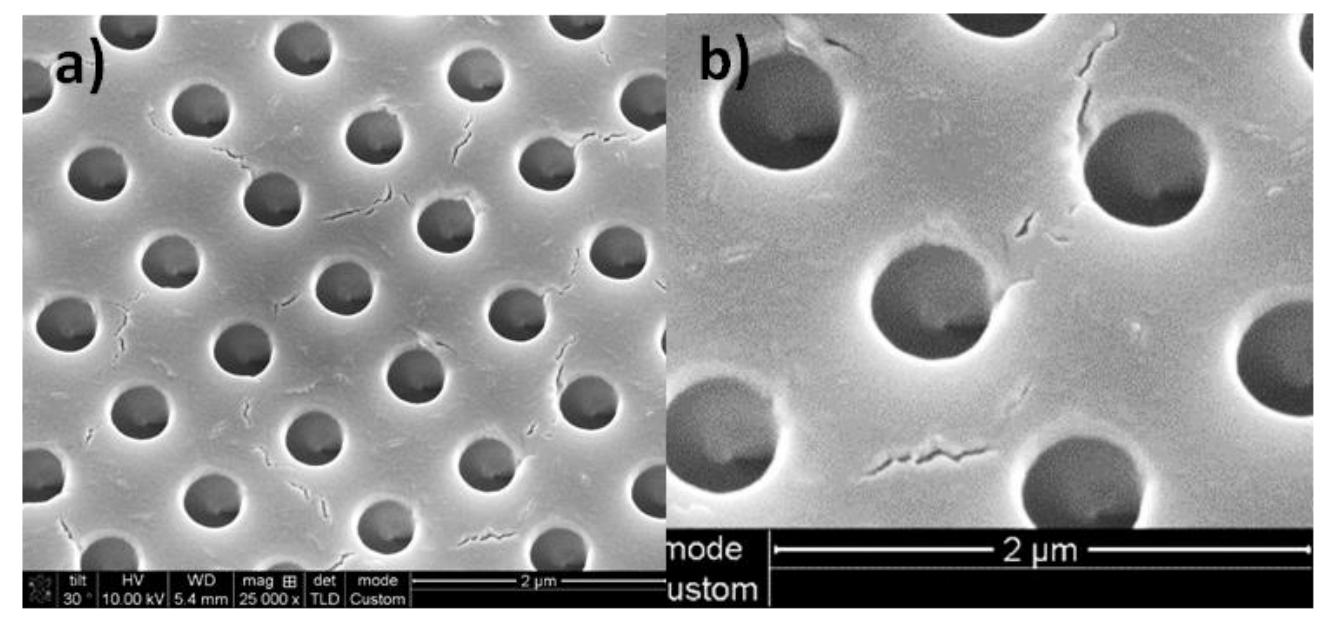

Figure 2: SEM image of $\mathrm{XeF}_{2}$ gas-assisted FIB etched $200 \mathrm{~nm}$ chiral circular array at a) low-magnification and $b$ ) high-magnification showing graded z-depth in the structure. Image was acquired at 15 degree tilt. 